Borra discusses, rather, first the moral canzoni, then the moral sonnets, then what he calls the "sonetti erotici," and finally the "canzoni amorose" (he distinguishes between the conventional tone of the pre-conversion canzoni, which he sees as "una poesia tutto sommato di maniera," and the parodic intent of the pre-conversion sonnets). I may seem overly fastidious in making this point, for the scribe, not the author, might have been responsible for the general ordering of the different sections (indeed, modern editors have tended to put the post-conversion canzoni later, after the lore canzoni, in order to recorer their ostensible order of composition). It also makes thematic sense for Borra to discuss all the post-conversion poems together, and then all the pre-conversion ones. But in this way he misses, in my opinion, some of the implications of the story that the codex actually tells - in which the entire love interlude functions as a sort of "flash-back" in the poet's implied biography - and the network of correspondences that helps to weave the opposing sections into a unitary macrotext.

As Borra points out, it is the fracture in the work, the division of the poetry into two rigorously separate sections that are attributed to two different personalities, Guittone and Frate Guittone, that constitutes the author's distinct signature and ironically furnishes the major narrative element tying the poems together. This observation bears comparison with some of the critical insights in John Freccero's ground-breaking book, Dante: The Poetics of Conversion. In general, Borra seems to have slighted North American criticism, which is somewhat surprising, since he holds an American Ph.D. and teaches in the United States. W'ith the vanity of all academics, I looked for an article of mine on Guittone (published in Modern Philology in 1997) in the bibliography and was naturally disappointed not to find it there, but its absence seems indicative. The book might have benefitted from a more solid placement within the context of Otd and New Medievalism in English. It fills an important niche, however, and will doubtless become the indispensable starting-place for any student of Guittone.

\title{
Olivia Holaies
}

Yale L'niversity

\section{Letizia Panizza, Sharon Wood Eds. A History of Women's Writing in Italy. Cambridge: Cambridge University Press, 2000, 361 pp.}

Le curatrici di A History' of II"omen's II"riting in Italy' seguono una tendenza diffusa ultimamente, a partire dall' ambiente accademico anglo-americano e poi anche italiano, di interesse per l'apporto letterario, ma anche culturale e sociale dato dalle donne in Italia. Su questa linea si collocano, ad esempio, testi quali Italian IVomen Writers, Feminist Eincyclopedia of ltalian I iternture (entrambe curate da Rinaldina Russell e pubblicate da Greenwood Press rispettivamente nel 1994 e 1997) o il saggio di Marina Zancan Il doppio itinerario della scrittura (Einaudi, 1998).

Tuttavia rispetto ai lavori finora pubblicati, di taglio specificamente bio-bi- 
bliografico, o limitati ad un periodo storico ristretto, o a poche autrici rappresentative, quest'opera si distingue per ampiezza, ricchezza, e per il fatto di essere un testo storiografico completo, ispirato a principi teorici femministi. A History of IV"omen's If riting in Italy è una storia della scrittura femminile dal Medioevo ai giorni nostri che rede la collaborazione di diciotto studiose d' italianistica europee e nordamericane. Un lavoro di queste dimensioni non era stato ancora tentato né in Italia né in altro paese di lingua inglese. Si tratta di un'opera collettiva di noterole portata, in cui le curatrici Letizia Panizza e Sharon IVood hanno compiuto un eccellente lavoro di coordinazione.

Nell'Introduzione Panizza e Wrood motivano il titolo e la preferenza per il termine scrittura anziché letteratura, quest'ultimo sentito come troppo limitante perché legato alle convenzionali distinzioni di genere letterario, poiché "Women's writing does not fit easily into literary tradition or canon," (1). Le donne scrittrici molto spesso non possederano quella preparazione formale basata sui classici e sulla tradizione latina. Anche scrittrici di noterole talento erano autodidatte e talvolta, non scrivendo nell' italiano standard renivano più facilmente escluse dal canone. I limiti geografici di questa Storia includono la scrittura femminile prodotta entro i confini territoriali dell'Italia, ma non escludono opere scritte in altre lingue, dal latino di Isotta Nogarola, al dialetto della poesia contemporanea di Franca Grisoni, all'inglese di AnnieVivanti, al francese di Giuseppina di Lorena Carignano, solo per dare alcuni esempi. Metodologicamente le curatrici rivelano la loro impronta femminista, anche se non intendono ghettizzare e separare la scrittura femminile dalla storia della letterartura italiana in generale. Panizza e Wood preferiscono non allinearsi con la nozione di écriture feminine, destinata a cadere in posizioni essenzialiste, tuttavia riconoscono l'importanza nella scrittura del genere/sesso, in quanto construtto sociale determinato da circostanze storiche. Le donne in Italia hanno sofferto per lungo tempo di limitato accesso all'alfabetizzazione e ai circoli intellettuali e culturali; non hanno riceruto la doruta attenzione nelle storie letterarie esistenti, perché la loro opera non si inseriva nei generi e negli stili sanciti dal canone tradizionale. Questa storia intende dunque dare una roce alla scrittura delle donne ed è in questo che si distingue dalle convenzionali storie della letteratura nazionale.

Il libro è strutturato in tre parti: I. "The Renaissance,Counter-Reformation and serenteenth century", II." The Enlightenment and Restoration", III. "The Risorgimento and modern ltaly, 1850-2000". La prima consiste di sei capitoli che includono scrittura epistolare di Maria Luisa Doglio, poesia in vernacolo e misteri teatrali di Judith Bryce, poesia lirica dal 1500 al 1650 di Gioranna Rabitt, fiction dal 1560 al 1650 di Virginia Cox, scritti polemici dal 1500 al 1650 di Letizia Panizza e letteratura religiosa-devozionale dal 1400 al 1600 di Gabriella Zarri. La seconda parte include quattro capitoli: uno sulla letteratura del diciottesimo secolo di Luisa Ricaldone, uno sulla scrittura di viaggio dal 1750 al 1860 di Ricciarda Ricorda, uno sul giornalismo dal 1750 al 1850 di V'erina Jones e infine uno su critiche letterarie e ricercatrici dal 1780 al 1850 di Adriana Chemello. Più estesa si presenta la terza ed ultima parte, con nove capitoli: uno di Silvana Patriarca su gionnalismo e saggistica dal 1850 al 1915, uno di Lucia Re su Futurismo e 
Fascismo dal 1914 al 1945, uno di Catherine ()'Brien su poesia dal 1870 al $20(0)$. Lo spazio più ampio è assegnato al romanzo a cui sono dedicati tre capitoli, che seguono un ordine cronologico dal 1850 al 1920 di Lucienne Kroha, dal 1945 al 1965 di Ann Hallamore Caesar, dal 1965 fino ai giorni nostri di Adalgisa Giorgio. Ottima e coerente la scelta di dedicare un capitolo a parte, di Inna Laura Lepschy; al romanzo popolare o d' appendice dal 1850 al 1920. In linea con l' intento indicato nell' introduzione, di dare spazio a coloro che più sono state marginalizzate, ottima e senza precedenti la scelta di assegnare un capitolo, di Aine ()' I lealy; al teatro e al cinema dal 1945 al 200(). ()Itre a donne già ben conosciute e studiate come Franca Rame, Iina Wertmuller o Liliana Cavani, si dà spazio a figure finora inedite come Titina De Filippo e Franca Valeri. Finalmente si dà il giusto riconoscimento al lavoro delle donne, in un ambito come quello dell'arte rappresentativa, in cui l'egemonia maschile troppo spesso offusca il contributo femminile.

La terza parte si conclude con un' altra novità rispetto alle storie letterarie tradizionali: il capitolo di Sharon IFood "Aesthetics and critical Theory:" Si introducono qui in maniera succinta, ma esauriente, le tendenze che hanno caratterizzato la critica femminista in Italia, dalla prima fase della doppia militanza (politica e femminismo), all'impegno teorico-filosofico, evidenziando la caratteristica oscillazione della critica femminista in Italia tra il modello d'astrazione teorico francese e quello pragmatico di impegno politico-sociale anglo-americano.

Non potendo in questa sede menzionare anche succintamente il contenuto di ciascun capirolo, ci limitiamo a dare un giudizio generale positivo. Ciascun capitolo si distingue per chiarezza ed essenzialità, mantenendo un perfetto equilibrio nelle proporzioni: dove nessun saggio prevale per estensione sugli altri e le note, pur ridotte al minimo, sono informative e ben compendiate dalla bibliografia finale. Un altro punto di forza del volume è la guida bibliografica (coordinata da Penny Morris) alle scrittrici e alle loro opere, che permette unagile e rapida consultazione.

Loderole l'impegno di riscoperta e recupero delle voci femminili rimaste sepolte nell' oblio che porta all' inclusione di generi come la scrittura epistolare, di viaggio, il giornalismo, l' appendicistica, il teatro e il cinema, la teoria femminista. forme generalmente marginalizzate o del tutto assenti dalle storie letterarie tradizionali.

Questo libro si distingue per novità, unicità, per essere dedicato esclusiramente alla scrittura delle donne, ma anche per la scelta per così dire 'ideologica' di includere saggi critici scritti esclusivamente da donne. Seguendo lo sviluppo della scrittura femminile in Italia, dalle sue stentate e difficili origini, fino ai giorni nostri, in cui per le donne scrittrici si prospetta un futuro di espansione e di successo, questo libro costruisce anche una suggestiva genealogia della scrittura femminile e afferma l'importanza e l'autorità ottenuta dalle donne. Come sostienc Wood a conclusione del saggio finale, il lavoro di riscoperta delle voci femminili continua e "promises not only to rewrite the history of Italian women's writing, but to reshape our reading of Italian literature itself." (280)

A History of II omen's II riting in Itaty colma un ruoto nell'ambito della storia 
letteraria italiana e costituisce uno strumento essenziale, non solo per tutti gli studiosi di scrittura femminile in Italia, ma anche per un pubblico non specializzato, semplicemente desideroso di informarsi sull' attività letteraria delle donne, troppo spesso assente dalla storiografia ufficiale. Ė riprovevole, ma forse significativo che, un lavoro di questa portata e importanza nel campo della scrittura femminile in Italia, venga scritto e pubblicato per primo in inglese e fuori dall' Italia.

\section{PITRIZIA BETTELLA \\ University of Alberta}

\section{Scorrano. Luigi. Il Dante "fascista". Saggi, letture, note dantesche. Raven- na: Longo, 2001. 209 pp.}

In un senso questo volume di Luigi Scorrano segue un modello veramente dantesco: si tratta di una collezione di saggi che discutono il testo dantesco insieme a saggi che trattano la fortuna di Dante nei secoli dopo la sua morte. Il Dante "fascista" rievoca non soltanto la famosa terza rima, in cui le rime guardano indietro mentre vanno avanti, ma anche la doppia prospettiva della Commedia - il retrosguardo del narratore in tensione con la progressione in avanti del pellegrino. Allo stesso tempo, la coesistenza nel volume della "lectura dantis" tradizionale e di saggi che considerano la presenza dantesca nella letteratura del Novecento sottolinea $\mathrm{i}$ due diversi indirizzi nel campo attuale degli studi danteschi. Scorrano, però, dimostra che tutte e due le prospettive, infatti, hanno un rapporto simbiotico e che si forniscono a vicenda di complementi adatti.

I tre primi saggi, presentati nella forma tradizionale della "lettura," dimostrano la profondità della sua comprensione del testo dantesco e delle questioni che esso produce. Il primo, intitolato "Inferno XIII: un orizzonte di negazione," considera la questione della negazione della possibilità di vivere trattata nell'episodio di Pier delle Vigne. Chiamando il consigliere di Stupor Mundi "l'anti-Catone," Scorrano affronta direttamente la questione problematica della differenza fra un rifiuto della vita in nome di una difesa della libertà e il gesto di disperazione di cui quello di Piero è un triste esempio. Quest'ultimo, dice Scorrano, nella sua negazione, rappresenta non soltanto il rifiuto della vita ma, più importante, il rifiuto del dono divino e, se si può estrapolare, di Dio.

Nella seconda delle "letture" ("Dall'abbandono alla bontà riconquistata (Purgatorio III)") lo studioso considera due topoi che si trovano dappertutto nella Commedia ma che trovano il loro apice negli ultimi canti del Purgatorio quando Dante si volge per scoprire che Virgilio è sparito. Le radici di questo momento e le sue prefigurazioni sono già presenti nei primi passi del pellegrino verso la vetta della montagna. II tema della fuga suggerisce l'abbandono di qualcosa mentre la presenza della montagna diventa quasi un personaggio verso cui il pellegrino corre. La montagna costituisce una specie di figura o almeno la manifestazione fisica della sfida spirituale che confronta i peccatori. Mentre la montagna, e speci- 